\title{
Proportionality in Approval-Based Elections With a Variable Number of Winners
}

\author{
Rupert Freeman $^{1}$, Anson Kahng ${ }^{2 *}$ and David M. Pennock ${ }^{3}$ \\ ${ }^{1}$ Microsoft Research New York City \\ ${ }^{2}$ Carnegie Mellon University \\ ${ }^{3}$ Rutgers University \\ rupert.freeman@microsoft.com, akahng@cs.cmu.edu,dpennock@dimacs.rutgers.edu
}

\begin{abstract}
We study proportionality in approval-based multiwinner elections with a variable number of winners, where both the size and identity of the winning committee are informed by voters' opinions. While proportionality has been studied in multiwinner elections with a fixed number of winners, it has not been considered in the variable number of winners setting. The measure of proportionality we consider is average satisfaction (AS), which intuitively measures the number of agreements on average between sufficiently large and cohesive groups of voters and the output of the voting rule. First, we show an upper bound on AS that any deterministic rule can provide, and that straightforward adaptations of deterministic rules from the fixed number of winners setting do not achieve better than a $1 / 2$ approximation to AS even for large numbers of candidates. We then prove that a natural randomized rule achieves a 29/32 approximation to AS.
\end{abstract}

\section{Introduction}

We study multiwinner approval-based elections, where a group of agents, or voters, selects a committee from a set of candidates based on the agents' preferences. Each agent expresses her preferences through an approval vote, where she designates a subset of candidates she approves for the committee, and all votes are then aggregated to select a winning committee from the pool of candidates.

Some multiwinner elections include a fixed committee size: the outcome must fill exactly $k$ seats on a committee. This is known as the fixed number of winners (FNW) setting, and there is a large body of work on the complexity and proportionality of various voting rules in the FNW setting [Aziz et al., 2017; Sánchez-Fernández et al., 2017; Aziz et al., 2018; Brill et al., 2017; Peters and Skowron, 2019; Skowron et al., 2017b]. In contrast, we are interested in the setting in which there is no a priori fixed committee size, also known as the variable number of winners (VNW) setting. In this case, both the size of the committee and the candidates chosen to sit on the committee are informed by agents' votes.

\footnotetext{
${ }^{*}$ Contact Author
}

We present a setting where VNW elections are a natural fit; Faliszewski et al. [2017] discuss others.

Consider an election that consists of a series of ballot measures, where each ballot question can easily be reversed such that "Yes" becomes "No" and "No" becomes "Yes". This is a practical concern, as ballots are often deliberately constructed such that a "Yes" on one question represents a vote in favor of upholding a current statute, while a "Yes" on another question down the ballot represents a vote in favor of repealing a current statute [Mueller, 1969]. In this case, voters derive utility from every decision they agree with, whether it is an approval vote or a disapproval vote. Note that, because there is no set number of measures that must be "elected" (i.e., passed), this constitutes a VNW election.

It can be important to ensure that the selected alternatives are chosen in a proportional manner. For instance, in the case of ballot measures, we may want to ensure that all groups in the electorate are satisfied with at least some of the outcomes. In other words, a small majority of the electorate should not be able to overrule a sizable minority on every ballot measure.

In order to study proportionality in FNW elections, researchers have proposed the axioms of justified representation (JR), proportional justified representation (PJR), extended justified representation (EJR), and average satisfaction (AS) [Aziz et al., 2017; Sánchez-Fernández et al., 2017], which capture the intuition that all sufficiently large groups that agree on sufficiently many candidates should achieve some measure of satisfaction. However, to our knowledge, we are the first to study representation in VNW elections.

Our Contributions. Our main research goal is to study proportionality in multiwinner elections with a variable number of winners. In particular, we study the proportionality measure of average satisfaction (AS) and show that there is a separation between the performance of deterministic and randomized voting rules.

As our first contribution, we develop a framework for thinking about proportionality in VNW elections. Previous work on proportionality in FNW elections is largely based on the concept of justified representation (and extensions thereof). However, as we discuss in Section 3, JR-based notions of proportionality are less compelling in VNW elections than in FNW elections. Therefore, we instead base our approach on the concept of average satisfaction, which is arguably a more robust version of justified representation. 
Second, in Section 4, we consider the proportionality guarantees of deterministic rules in the VNW setting. We extend three existing deterministic rules for the FNW setting to the VNW setting, and show that these rules do not guarantee good approximations to average satisfaction. We also prove upper bounds on the level of average satisfaction that any deterministic rule can provide.

Finally, in Section 5, motivated by the shortcomings of deterministic rules, we turn our attention to randomized rules and show that a natural randomized rule provides a good approximation to average satisfaction.

\subsection{Related Work}

There is a significant body of work studying proportionality in FNW elections. As mentioned above, Aziz et al. [2017] put forward the compelling axiom of justified representation (JR), as well as a stronger version of this axiom, extended justified representation (EJR) to capture the notion that any sufficiently large and cohesive group of voters deserves some measure of representation in the elected committee. SánchezFernández et al. [2017] build on this idea by introducing the intermediate axiom of proportional justified representation (PJR), a relaxation of EJR that is more stringent than JR.

Average satisfaction (AS) was first defined by SánchezFernández et al. [2017], who study the average satisfaction guaranteed by extended justified representation (EJR). Further work by Aziz et al. [2018] shows that Proportional Approval Voting (PAV) guarantees a level of average satisfaction that implies EJR. Additionally, Skowron et al. [2017a] extend the notion of average satisfaction to the context of complete rankings as opposed to committee selection. Further work by Skowron [2018] studies the proportionality degree of various multiwinner rules by considering the average satisfaction of all groups of a certain size.

There is also a significant body of work studying VNW elections; however, to the best of our knowledge, none of the proposed rules satisfy proportionality (and, in general, that is not their goal). Kilgour [2016] proposes a multitude of rules for VNW elections, including satisfaction approval voting and variants thereof. In a related vein, Kilgour et al. [2006] and Brams et al. [2007] study the minimax and minisum rules for selecting a committee in the VNW setting. Fishburn and Pekeč [2004] study threshold approaches to committee selection, which are VNW rules in the sense that the size of the selected committee depends on the approval votes. Additionally, the Mean Rule [Duddy et al., 2016] and Borda Mean Rule [Brandl and Peters, 2019] can be seen as VNW rules when given approval votes. Finally, Faliszewski et al. [2017] study the computational complexity of various VNW rules, but do not consider proportionality in their analysis.

\section{Preliminaries}

Let $N=\left\{v_{1}, \ldots, v_{n}\right\}$ be a set of $n$ voters and $C=$ $\left\{c_{1}, \ldots, c_{m}\right\}$ be a set of $m$ candidates. For every voter $v_{i}$, denote by $A_{i} \subseteq C$ the set of candidates that are approved by $v_{i}$. A preference profile $\mathbf{A}=\left\{A_{1}, \ldots, A_{n}\right\}$ is the set of all voter preferences $A_{i}$.
A variable number of winners (VNW) voting rule $f$ takes as input a preference profile $\mathbf{A}$ and outputs some set of candidates $f(\mathbf{A}) \subseteq C$. Note that we allow $f(\mathbf{A})=\emptyset$ or $f(\mathbf{A})=C$. We will also consider randomized VNW voting rules that output a distribution over sets of candidates.

Throughout this paper, we will denote by $W$ the set of candidates included in the committee, and we will denote by $C \backslash W$ the set of candidates excluded from the committee.

We say that a group of voters $V \subseteq N$ is $\ell$-large if $|V| \geq$ $\ell \cdot \frac{n}{m}$, and $\ell$-cohesive if $\left|\cap_{i \in V} A_{i}\right|+\left|\cap_{i \in V} C \backslash A_{i}\right| \geq \ell$. We will also say that a group of voters $V$ agrees on a candidate $c_{j}$ if $c_{j} \in A_{i}$ for all $i \in V$ or $c_{j} \notin A_{i}$ for all $i \in V$. Otherwise, we say that $V$ disagrees on $c_{j}$. Intuitively, a group of voters is $\ell$-large and $\ell$-cohesive if they constitute an $\ell / m$ fraction of all voters who agree on $\ell$ out of $m$ candidates.

In our work, we consider a different measure of representation than in the FNW setting. In the FNW setting, voters derive utility from the number of their approved candidates elected to the committee. However, this definition cannot be easily adapted to the VNW setting because then a rule could maximally satisfy all voters by including all candidates on the committee. Therefore, we assume that voters derive utility from agreeing with the placement of candidates either on the committee or not on the committee. For instance, in an election with two candidates, $c_{1}$ and $c_{2}$, if a voter $i$ has approval set $A_{i}=\left\{c_{1}\right\}$ (i.e., she approves $c_{1}$ and disapproves $c_{2}$ ), then she receives one unit of utility for the output committee $\left\{c_{1}, c_{2}\right\}$ because she agrees with the inclusion of $c_{1}$ but disagrees with the inclusion of $c_{2}$.

With this in mind, the following definition of average satisfaction is adapted from the definition of Sánchez-Fernández et al. [2017] in the FNW setting.

Definition 1. Given a set of candidates $W \subseteq C$, the average satisfaction of a group of voters $V \subseteq N$ is

$$
\operatorname{avs}_{W}(V)=\frac{1}{|V|} \sum_{i \in V}\left(\left|A_{i} \cap W\right|+\left|\left(C \backslash A_{i}\right) \cap(C \backslash W)\right|\right) .
$$

We can now define AS in the VNW setting. ${ }^{1}$ The intuition behind the following definition is that any sufficiently large and cohesive group of voters deserves to be adequately represented on average, which is a departure from justified representation-based axioms that have been studied in the FNW setting. Intuitively, JR-like notions of proportionality only require that some member of each cohesive group is represented to some extent, whereas average satisfaction requires all members of each cohesive group to be represented (at least on average).

Definition 2. A set of candidates $W \subseteq C$ satisfies $\alpha$-AS if, for all $\ell$-large and $\ell$-cohesive groups of voters $V \subseteq N$, $\operatorname{avs}_{W}(V) \geq \alpha \cdot \ell$ for all $\ell \in[m]$. For brevity, we refer to the special case of 1-AS as AS.

The following example demonstrates cohesiveness and average satisfaction.

\footnotetext{
${ }^{1}$ Note that we overload the use of the term "average satisfaction" to refer to both the numerical quantity from Definition 1 (average satisfaction) as well as the axiomatic property in Definition 2 (AS).
} 
Example 1. Consider the following profile with $n=8$ voters, $v_{1}, \ldots, v_{8}$, and $m=4$ candidates, $c_{1}, \ldots, c_{4}$, with preferences

$$
\begin{aligned}
A_{1}=A_{2} & =\left\{c_{1}, c_{2}, c_{3}, c_{4}\right\} & & A_{6}=\left\{c_{2}, c_{3}\right\} \\
A_{3}=A_{4} & =\left\{c_{1}, c_{2}\right\} & A_{7} & =\left\{c_{3}\right\} \\
A_{5} & =\left\{c_{1}, c_{3}\right\} & A_{8} & =\left\{c_{4}\right\} .
\end{aligned}
$$

Now, consider the output $W=\left\{c_{4}\right\}$. Note that each voter agrees with the output on the placement of at least one candidate, so for any 1-large and 1-cohesive group $V_{(1)}$ (i.e., a group of $1 \cdot \frac{n}{m}=2$ voters who agrees on the placement of 1 candidate), avs ${ }_{W}\left(V_{(1)}\right) \geq 1$. Furthermore, note that there is only one 2-large and 2-cohesive group of voters: $v_{1}, v_{2}, v_{3}$, and $v_{4}$ agree on the placement of $c_{1}$ and $c_{2}$, but disagree on the placement of $c_{3}$ and $c_{4}$, so they constitute a 2-large group of voters who agree on 2 candidates. Let $V_{(2)}=\left\{v_{1}, v_{2}, v_{3}, v_{4}\right\}$. Note that avs $s_{W}\left(V_{(2)}\right)=1$ because each $v \in V$ agrees with $W$ on exactly one placement, but because this group of voters is 2-large and 2-cohesive, we see that $W$ only satisfies $1 / 2-A S$ in this scenario.

Given our definition of voter satisfaction, we can straightforwardly extend the following deterministic multiwinner rules from the FNW setting to the VNW setting.

Proportional Approval Voting (PAV). Under the PAV rule [Thiele, 1895], voter $i$ derives utility $H_{k}=1+1 / 2+$ $\cdots+1 / k$ from a committee $W$, where $k=\left|A_{i} \cap W\right|+\mid(C \backslash$ $\left.A_{i}\right) \cap(C \backslash W) \mid$ is the number of candidate placements that $i$ agrees with. The goal of PAV is to maximize the sum of all voters' utilities, and thus PAV outputs the subset $W \subseteq C$ with highest PAV-score.

Sequential Phragmén (seq-Phragmén). The seqPhragmén rule [Phragmén, 1899; Janson, 2016; Brill et al., 2017] is defined as follows. Each candidate carries a load of one unit, and this load is distributed among voters who agree with the placement of this candidate in either the included set or excluded set. The seq-Phragmén rule proceeds iteratively by, in each round, placing the candidate that results in the smallest increase in the maximal load of any voter.

Let $x_{i}^{(t)}$ denote the load of voter $i$, and $s^{(t)}$ the maximal load, after $t$ candidates have been placed. All voters start out with no load, $x_{i}^{(0)}=0$. Furthermore, let $N_{j}=\{i \in$ $\left.N: c_{j} \in A_{i}\right\}$ represent the set of voters that approve of candidate $c_{j}$. The maximal voter load if, on the $t^{t h}$ placement, candidate $c_{j}$ is included in the committee is

$$
s^{(t)}\left(c_{j}\right)=\frac{1+\sum_{i \in N_{j}} x_{i}^{(t-1)}}{\left|N_{j}\right|},
$$

and the maximal voter load if candidate $c_{j}$ is excluded from the committee is

$$
s^{(t)}\left(\overline{c_{j}}\right)=\frac{1+\sum_{i \in N \backslash N_{j}} x_{i}^{(t-1)}}{\left|N \backslash N_{j}\right|}
$$

because the load is distributed so as to equalize the loads of all voters who agree with the placement of $c_{j}$. At each step $t$, seq-Phragmén places the candidate $c_{j}$ that minimizes $\min \left(s^{(t)}\left(c_{j}\right), s^{(t)}\left(\overline{c_{j}}\right)\right)$ and updates voter loads accordingly: in the case that $c_{j}$ is included in the committee,

$$
x_{i}^{(t)}= \begin{cases}s^{(t)}\left(c_{j}\right) & \text { if } i \in N_{j} \\ x_{i}^{(t-1)} & \text { otherwise }\end{cases}
$$

and in the case that $c_{j}$ is excluded from the committee,

$$
x_{i}^{(t)}= \begin{cases}s^{(t)}\left(\overline{c_{j}}\right) & \text { if } i \in N \backslash N_{j} \\ x_{i}^{(t-1)} & \text { otherwise. }\end{cases}
$$

This rule proceeds until all candidates have been placed, and then returns the included and excluded candidates.

Rule X. Rule X [Peters and Skowron, 2019] allocates each voter a budget of one dollar, which they then spend on placing candidates either in the included set or excluded set. Placing a candidate costs $n / m$ dollars, and the set of voters who agree on the placement of this candidate must be able to collectively afford the placement. The rule starts with an empty included set $W$ and an empty excluded set $\bar{W}$, and it iteratively places candidates in the committee or its complement as follows.

Let $b_{i}(t)$ be the amount of money that voter $i$ has remaining after the $t^{t h}$ candidate is placed; i.e., $b_{i}(0)=1$ for all voters $v_{i} \in N$. At the $t^{t h}$ step, we say that a candidate $c \notin W \cup \bar{W}$ is $q$-affordable for some $q \geq 0$ if

$$
\max \left(\sum_{i: c \in A_{i}} \min \left(q, b_{i}(t-1)\right), \sum_{i: c \in C \backslash A_{i}} \min \left(q, b_{i}(t-1)\right)\right) \geq n / m .
$$

In other words, candidate $c$ is $q$-affordable if it can be placed in either the included or excluded set while voters who approve or disapprove of $c$ each pay a maximum of $q$ dollars. If no candidate is $q$-affordable for any $q \geq 0$, then the rule stops, placing the current set of included candidates into $W$, the current set of excluded candidates into $C \backslash W$, and placing arbitrarily any candidates not already put into $W$ or into $C \backslash W$. Else, the rule places the candidate which is $q$ affordable for the minimum value $q$ in the approved or disapproved committee, according to voter preferences. Each voter who agrees with this placement has their budget updated to $b_{i}(t)=b_{i}(t-1)-\min \left(q, b_{i}(t-1)\right)$, and the process continues.

\section{Justified Representation in VNW Elections}

In order to build intuition about why we focus on AS instead of (E/P)JR, we begin by defining JR, PJR, and EJR for VNW elections. In each case, the definition is a straightforward adaptation of the corresponding definition for the FNW setting, where we intuitively replace "agreement with members on the committee" with "agreement on the placement of each candidate." We slightly overload notation-namely, JR, PJR, and E JR-from the FNW setting in the following definitions.

Definition 3 (JR). Consider a ballot profile A. A set of candidates $W \subseteq C$ satisfies justified representation (JR) with respect to $\mathbf{A}$ if, for all sets of 1-large and 1-cohesive voters $N^{*}$, there exists an $i \in N^{*}$ such that $\left|A_{i} \cap W\right|+\mid\left(C \backslash A_{i}\right) \cap$ $(C \backslash W) \mid \geq 1$. 
Definition 4 (PJR). Consider a ballot profile A. A set of candidates $W \subseteq C$ satisfies proportional justified representation (PJR) with respect to $\mathbf{A}$ if, for all $\ell$-large and $\ell$-cohesive groups of voters $N^{*},\left|\bigcup_{i \in N^{*}} A_{i} \cap W\right|+\mid\left(\bigcup_{i \in N^{*}}\left(C \backslash A_{i}\right)\right) \cap$ $(C \backslash W) \mid \geq \ell$ for all $\ell \in[m]$.

Definition 5 (EJR). Consider a ballot profile A. A set of candidates $W \subseteq C$ satisfies extended justified representation (EJR) with respect to $\mathbf{A}$ if, for all $\ell$-large and $\ell$-cohesive groups of voters $N^{*}$, there exists an $i \in N^{*}$ such that $\left|A_{i} \cap W\right|+\left|\left(C \backslash A_{i}\right) \cap(C \backslash W)\right| \geq \ell$ for all $\ell \in[m]$.

The following example illustrates these definitions.

Example 2. Consider the same profile as in Example 1 with $n=8$ voters, $v_{1}, \ldots, v_{8}$, and $m=4$ candidates, $c_{1}, \ldots, c_{4}$.

Again, consider the output $W=\left\{c_{4}\right\}$. W satisfies JR because each voter agrees with the output on the placement of at least one candidate. Furthermore, $W$ satisfies PJR because, on the only 2-large and 2-cohesive group of voters, $\left\{v_{1}, v_{2}, v_{3}, v_{4}\right\}$, two of them agree with the placement of $c_{3}$ and two of them agree with the placement of $c_{4}$. However, $W$ does not satisfy EJR because no voter in the coalition agrees with two placements of $W$-they all agree with exactly one placement.

We also study the relationship between the extensions of PAV, seq-Phragmén, and Rule X, and different notions of justified representation in the VNW setting. The proofs of the following propositions are omitted due to space constraints. ${ }^{2}$

Proposition 1. PAV satisfies PJR.

Proposition 2. Seq-Phragmén satisfies PJR.

Proposition 3. Rule X satisfies PJR but not EJR.

Notably, in the VNW setting, JR and PJR are less compelling notions of representation than in the FNW setting. In particular, whenever an $\ell$-cohesive group of voters does not agree on the placement of a particular candidate, PJR automatically counts that candidate toward the group's representation quota, since at least one member of the group agrees with the candidate's placement. In other words, any disagreement within an $\ell$-cohesive group results in partial representation, no matter the outcome of the election. This is particularly problematic for JR: any 1-large, 1-cohesive group of voters that disagrees on even a single candidate will never be witness to a violation of JR.

Proposition 3 is also notable because Rule X satisfies EJR for FNW elections, but the straightforward extension of this rule does not satisfy EJR for VNW elections, demonstrating a qualitative difference between proportionality properties in the FNW and VNW settings. It is still an open question whether or not PAV and seq-Phragmén satisfy EJR for VNW elections.

\section{Deterministic Rules}

We begin by showing an upper bound on the level of average satisfaction that deterministic rules can provide.

\footnotetext{
${ }^{2}$ All omitted proofs can be found in the full version of the paper on the authors' websites.
}

Theorem 1. No deterministic rule satisfies $\left(\frac{m-1}{m}+\epsilon\right)$-AS for any $m$ and any $\epsilon>0$.

Proof. First, suppose that $m$ is odd. Then set $n=2$, with $A_{1}=\left\{c_{1}, \ldots, c_{m}\right\}$ and $A_{2}=\emptyset$. Without loss of generality, suppose that the output $W$ is such that $|W|>\frac{m}{2}$. But then voter $v_{2}$ is an $\frac{m}{2}$-large, $\frac{m}{2}$-cohesive group with average satisfaction at most $\frac{m-1}{2}$, which yields an $\left(\frac{m-1}{m}\right)$-AS approximation.

Next, suppose $m$ is even, and set $n=4 m$. Consider the profile

$$
\begin{array}{ll}
A_{1}=\left\{c_{1}, \ldots, c_{m}\right\} & A_{3}=\left\{c_{m}\right\} \\
A_{2}=\left\{c_{1}, \ldots, c_{m-1}\right\} & A_{4}=\emptyset
\end{array}
$$

Again, without loss of generality, suppose that the output $W$ is such that $|W| \geq \frac{m}{2}$. We consider two cases. In the first case, suppose that the output $W$ has $|W| \geq \frac{m}{2}+1$. Consider the $\frac{m}{2}$-large, $\frac{m}{2}$-cohesive group of voters $V \stackrel{2}{=}\left\{v_{3}, v_{4}\right\}$. We have

$$
\operatorname{avs}_{W}(V) \leq \frac{1}{2}(m-|W|+m-|W|+1) \leq \frac{m-1}{2}
$$

which yields at most an $\left(\frac{m-1}{m}\right)$-AS approximation.

In the second case, suppose that the output $W$ has $|W|=$ $\frac{m}{2}$. Suppose that $c_{m} \notin W$ (the case of $c_{m} \in W$ follows symmetrically). Then again consider $V=\left\{v_{3}, v_{4}\right\}$. We have

$$
\operatorname{avs}_{W}(V) \leq \frac{1}{2}\left(\frac{m}{2}-1+\frac{m}{2}\right)=\frac{m-1}{2}
$$

again yielding an $\left(\frac{m-1}{m}\right)$-AS approximation. This completes the proof.

Theorem 1 leaves open the possibility that there exists a deterministic rule that provides quite good average satisfaction guarantees when the number of candidates is large. Finding such a rule or lowering the upper bound is an interesting open question. However, we show that none of the natural adaptations of FNW rules that we consider is able to guarantee better than a 0.5 approximation to AS even when $m$ is large.

Theorem 2. PAV does not satisfy $(0.5+\epsilon)-A S$, for any $\epsilon>0$ for $m \geq 2$.

Proof. Consider a profile with $n=2 m$ voters with preferences

$$
\begin{aligned}
A_{1}=\ldots=A_{m-1} & =\left\{c_{1}, \ldots, c_{m}\right\} \\
A_{m}=\ldots=A_{2 m-2} & =\left\{c_{1}, \ldots, c_{m-1}\right\} \\
A_{2 m-1} & =\left\{c_{m}\right\} \\
A_{2 m} & =\emptyset .
\end{aligned}
$$

This profile is symmetric in $c_{m}$, so without loss of generality suppose that $c_{m}$ is included. Suppose that some $k-1<$ $m-1$ of the candidates $c_{1}, \ldots, c_{m-1}$ are included. Then, the change in PAV score that would result from including an additional candidate is

$$
\begin{aligned}
\frac{m-1}{k+1} & +\frac{m-1}{k}-\frac{1}{m-k}-\frac{1}{m-k+1} \\
& \geq \frac{m-1}{m}+1-1-\frac{1}{2} \geq 0,
\end{aligned}
$$


where the first inequality holds because $k<m$.

Therefore, the maximum PAV score is achieved when all candidates $c_{1}, \ldots, c_{m-1}$ are included. But then the group $N^{*}=\left\{v_{2 m-1}, v_{2 m}\right\}$ is 1-large and 1-cohesive but is only satisfied 0.5 times on average.

Theorem 3. seq-Phragmén does not satisfy $(0.5+\epsilon)$-AS, for any $\epsilon>0$ for $m \geq 2$.

Proof. Consider the same profile as in the proof of Theorem 2. It is easy to check that seq-Phragmén begins by including candidates $c_{1}, \ldots, c_{m-2}$, after which each voter of the first and second type has load $\frac{m-2}{2(m-1)}$. In the $(m-1)$-th round, the algorithm has four choices: to include or exclude $c_{m-1}$, or to include or exclude $c_{m}$.

Including $c_{m-1}$ results in a load of $\frac{m-1}{2(m-1)}=\frac{1}{2}$ on voters $v_{1}, \ldots, v_{2 m-2}$. Excluding $c_{m-1}$ results in a load of $\frac{1}{2}$ to voters $v_{2 m-1}$ and $v_{2 m}$. Including $c_{m}$ (which is symmetric to excluding $c_{m}$ ) results in a load $x$ to voters $v_{1}, \ldots, v_{m-1}, v_{2 m-1}$, where $x$ is the solution to $m x-(m-1) \frac{m-2}{2(m-1)}=1$, which yields a solution of $x=\frac{1}{2}$.

The algorithm is therefore indifferent between all possible actions; breaking ties adversarially yields the inclusion of $c_{m-1}$. Regardless of the inclusion or exclusion of candidate $c_{m}$, the group $N^{*}=\left\{v_{2 m-1}, v_{2 m}\right\}$ is 1-large and 1-cohesive but is only satisfied 0.5 times on average.

We note that the dependence on tiebreaking in the proof of Theorem 3 can be removed by taking multiple copies of the profile used in the proof and changing the preference of a single voter.

Theorem 4. Rule $X$ does not satisfy $(0.5+\epsilon)-A S$, for any $\epsilon>0$ for $m \geq 3$. $^{3}$

Proof. Consider the same profile used in the proof of Theorem 2. Rule $\mathrm{X}$ begins by including each of candidates $c_{1}, \ldots, c_{m-1}$. Each of these candidates costs $\frac{n}{m(2 m-2)}=$ $\frac{1}{m-1}$ for each voter $v_{1}, \ldots, v_{2 m-2}$. In comparison, placing the last candidate at any point costs $n / 2$ voters $\frac{n / m}{n / 2}=\frac{2}{m}$, which is a greater cost than $\frac{1}{m-1}$ when $m \geq 3$. Including each of $c_{1}, \ldots, c_{m-1}$ therefore costs $v_{1}, \ldots, v_{2 m-2}$ one dollar each. Regardless of the placement of $c_{m}$, the 1-large and 1-cohesive group of voters $N^{*}=\left\{v_{2 m-1}, v_{2 m}\right\}$ is satisfied only 0.5 times on average.

\section{Randomized Rules}

We now turn our attention to randomized rules in order to achieve better average satisfaction guarantees. A randomized rule is one that outputs a distribution over committees rather than a single committee, and our approximation guarantee will hold in expectation over the possible committees. ${ }^{4}$ We consider a simple and natural randomized rule that, for each candidate $c_{j}$, includes $c_{j}$ in the set of winners $W$ with

\footnotetext{
${ }^{3}$ When $m=2$, we know from Theorem 1 that no deterministic rule, including Rule X, can achieve better than a 0.5 approximation.

${ }^{4}$ Recent work by Cheng et al. [2019] has applied randomization to proportionality in the FNW setting as well.
}

probability equal to the fraction ${ }^{5}$ of the voters who approve $c_{j}$

Definition 6. Given a preference profile $\mathbf{A}$, the Proportional Random Rule (PRR) independently includes each $c_{j} \in C$ in the winning committee $W$ with probability

$$
p_{j}=\frac{\mid\left\{v_{i} \in N \text { s.t. } c_{j} \in A_{i}\right\} \mid}{n} .
$$

Theorem 5. PRR satisfies $29 / 32-A S$ in expectation for any $m$.

In the proof of Theorem 5, it will be helpful to think about the effect that an individual candidate has on the satisfaction of a group $G$. For an outcome $W$, a group of voters $G$, and a candidate $c_{j}$, we say that the contribution from $c_{j}$ to the average satisfaction of $G$ is avs $s_{c_{j}}(G)=\left|\left\{i: c_{j} \in A_{i}\right\}\right| /|G|$ if $c_{j} \in W$ or $\operatorname{avs}_{c_{j}}(G)=\left|\left\{i: c_{j} \notin A_{i}\right\}\right| /|G|$ if $c_{j} \notin W$. Note that $\operatorname{avs}_{W}(G)=\sum_{j=1}^{m}$ avs $_{c_{j}}(G)$.

Proof. We prove the result in two steps. First, we show that when $\ell \leq m / 3$, PRR achieves an average satisfaction of $\ell$; second, we show that when $\ell>m / 3$, PRR achieves an average satisfaction of $(29 / 32) \ell$.

Case 1: $\ell \leq m / 3$. Consider an $\ell$-cohesive group, $G$, of size $\ell n / m$, and a candidate $c_{j}$. Note that it is sufficient to consider groups of size exactly $\ell n / m$ because if there exists an $\ell$-cohesive larger group that violates the desired guarantee, there must exist a subset of size $\ell n / m$ that also violates the guarantee. Let $k_{A}=\left|\left\{v_{i} \in G: c_{j} \in A_{i}\right\}\right|$ denote the number of voters in $G$ who approve $c_{j}$, and $k_{D}=\ell n / m-k_{A}$ denote the number of voters in $G$ who disapprove $c_{j}$. Without loss of generality, let $k_{A} \leq k_{D}$. Further, suppose that $x$ of the voters in $N \backslash G$ approve $c_{j}$ and $y=n-\ell n / m-x$ voters in $N \backslash G$ disapprove $c_{j}$.

The expected contribution from $c_{j}$ to the average satisfaction of $G$ is

$$
\mathbb{E}\left[\operatorname{avs}_{c_{j}}(G)\right]=\frac{k_{A}}{|G|}\left(\frac{k_{A}+x}{n}\right)+\frac{k_{D}}{|G|}\left(\frac{k_{D}+y}{n}\right) .
$$

Because $k_{A} \leq k_{D}$ and $x+y$ is fixed, this expression is minimized when $y=0$. We therefore have

$$
\begin{aligned}
\mathbb{E}\left[\operatorname{avs}_{c_{j}}(G)\right] & \geq \frac{k_{A}}{|G|}\left(\frac{k_{A}+n-\ell n / m}{n}\right)+\frac{k_{D}}{|G|}\left(\frac{k_{D}}{n}\right) \\
& =\frac{1}{n|G|}\left(|G|^{2}+k_{A}\left(n-\ell n / m-2 k_{D}\right)\right) \\
& \geq \frac{|G|}{n}=\frac{\ell}{m},
\end{aligned}
$$

where the inequality holds because $k_{D} \leq \ell n / m$ by definition, and we can assume $m \geq 3$ because $\ell$ must be at least 1 . Summed over all candidates, the average satisfaction of $G$ is at least $\ell$, as required.

\footnotetext{
${ }^{5}$ The marginal probabilities for each candidate being included in the committee are the same under this rule as the random dictatorship rule. The distribution over committees induced by the two rules is different, however.
} 
Case 2: $\ell>m / 3$. Consider an $\ell$-cohesive group, $G$, of size $\ell n / m$, and a candidate $c_{j}$. Let $k_{A}=\mid\left\{v_{i} \in G: c_{j} \in\right.$ $\left.A_{i}\right\} \mid$ denote the number of voters in $G$ who approve $c_{j}$, and $k_{D}=\ell n / m-k_{A}$ denote the number of voters in $G$ who disapprove $c_{j}$. Without loss of generality, let $k_{A} \leq k_{D}$. As in the previous case, it is easy to show that the expected contribution from $c_{j}$ to $G$ 's average satisfaction is minimized when all voters in $N \backslash G$ approve $c_{j}$.

We therefore have that

$$
\mathbb{E}\left[\operatorname{avs}_{c_{j}}(G)\right]=\frac{k_{A}}{|G|}\left(\frac{k_{A}+n-\ell n / m}{n}\right)+\frac{k_{D}}{|G|}\left(\frac{k_{D}}{n}\right) .
$$

Substituting $k_{D}=\ell n / m-k_{A}$, taking the derivative with respect to $k_{A}$, and setting to 0 yields

$$
\frac{1}{n}\left(4 k_{A}-3(\ell n / m)+n\right)=0 \Longrightarrow k_{A}=\frac{3 \ell n / m-n}{4}>0 \text {, }
$$

where the inequality follows from the assumption that $\ell>$ $m / 3$. Furthermore, the second derivative with respect to $k_{A}$ is $4 / n>0$, and therefore $k_{A}=(3 \ell n / m-n) / 4$ is a local minimum.

The expected contribution from $c_{j}$ to $G$ 's average satisfaction can therefore be as low as

$$
\begin{aligned}
\mathbb{E}\left[\operatorname{avs}_{c_{j}}(G)\right] & =\frac{k_{A}}{|G|}\left(\frac{k_{A}+n-\ell n / m}{n}\right)+\frac{k_{D}}{|G|}\left(\frac{k_{D}}{n}\right) \\
& =\frac{-\ell}{8 m}+\frac{3}{4}-\frac{m}{8 \ell} .
\end{aligned}
$$

We also note that, because $G$ is $\ell$-cohesive, there exist at least $\ell$ candidates that $G$ agrees on. Each of these candidates has

$$
\operatorname{avs}_{c_{j}}(G) \geq|G| / n \geq \ell / m,
$$

where the first inequality follows from $G$ being $\ell$-cohesive and the second from $G$ being $\ell$-large.

Summing over the contributions of all candidates, the average satisfaction of $G$ is at least

$$
\begin{aligned}
& \ell \frac{\ell}{m}+(m-\ell)\left(\frac{3}{4}-\frac{\ell}{8 m}-\frac{m}{8 \ell}\right) \\
& =\left(\frac{9 \ell}{8 m}-\frac{m^{2}}{8 \ell^{2}}-\frac{7}{8}+\frac{7 m}{8 \ell}\right) \ell .
\end{aligned}
$$

Our goal is to lower bound the term in parentheses by $\frac{29}{32}$, thus providing the desired approximation guarantee. Setting $\ell=\alpha m$, where $\alpha \in\left(\frac{1}{3}, 1\right)$, and differentiating with respect to $\alpha$ yields

$$
\frac{d}{d \alpha}\left(\frac{9 \alpha}{8}-\frac{1}{8 \alpha^{2}}-\frac{7}{8}+\frac{7}{8 \alpha}\right)=\frac{9}{8}+\frac{2}{8 \alpha^{3}}-\frac{7}{8 \alpha^{2}} .
$$

Setting equal to 0 yields

$$
9 \alpha^{3}-7 \alpha+2=(1+\alpha)(3 \alpha-2)(3 \alpha-1)=0,
$$

so the only critical point in the interval $\alpha \in(1 / 3,1]$ is $\alpha=2 / 3$. It is easy to check that the second derivative is positive at $\alpha=2 / 3$, so average satisfaction is minimized at this point. Plugging $\ell=2 m / 3$ into Equation 1 yields a 29/32 approximation to $\mathrm{AS}$, as desired.
Guided by Theorem 5, we show that the bound is tight.

Theorem 6. PRR does not satisfy $(29 / 32+\epsilon)-A S$ for any $\epsilon>0$.

Proof. Let $m=3$ and $n=12$. Consider the profile

$$
\begin{aligned}
A_{1}=A_{2}=A_{3}=A_{4}=A_{5} & =\left\{c_{1}, c_{2}, c_{3}\right\} \\
A_{6}=A_{7}=A_{8} & =\left\{c_{1}, c_{2}\right\} \\
A_{9}=A_{10}=A_{11}=A_{12} & =\emptyset .
\end{aligned}
$$

In particular, note that the first 8 voters form a 2-large and 2-cohesive group. Then the expected satisfaction of the first five voters is $\frac{2}{3}+\frac{2}{3}+\frac{5}{12}=\frac{21}{12}$ and the expected satisfaction of the next three voters is $\frac{2}{3}+\frac{2}{3}+\frac{7}{12}=\frac{23}{12}$. Taking the average yields $\frac{1}{8}\left(5 \frac{21}{12}+3 \frac{23}{12}\right)=\frac{29}{16}=\frac{29}{32} \ell$ for $\ell=2$.

Whether there exists a randomized rule that achieves better than a 29/32-AS approximation remains an open problem.

Before concluding this section, we note a final interesting and desirable property of PRR: strategyproofness. Since decisions are made on each candidate independently, voters maximize their expected satisfaction by reporting their true approval preferences.

\section{Conclusion}

We have initiated the study of representation in approval elections with a variable number of winners. We believe that this topic, and the study of VNW elections more generally, deserves further research.

Many open problems remain. In particular, we do not have matching upper and lower bounds for the average satisfaction guarantees that can be provided by deterministic and randomized rules. Determining the existence of rules that satisfy EJR is also an interesting question; while we have argued that natural extensions of JR and PJR make less sense for VNW elections than for FNW, EJR remains a compelling property.

More broadly, we have assumed that voters gain utility whenever they agree with the placement of a candidate, either included or excluded. This is a natural model when the notions of inclusion and exclusion are symmetric, as in the ballot measure example. In other settings it makes sense to consider other utility models. For instance, a natural extension of our model would consider voters who derive different levels of utility for an approved candidate being selected and a disapproved candidate being excluded, or even negative utility from an approved candidate not being selected or a disapproved candidate being included. The latter utility model is reminiscent of rules such as net satisfaction approval voting (NSAV) [Kilgour and Marshall, 2012], and precision and recall metrics in information retrieval. Extending our results to this setting appears nontrivial.

\section{Acknowledgments}

The majority of this work was done while David M. Pennock was at Microsoft Research New York City, and Anson Kahng was a research intern at Microsoft Research New York City. We also thank our anonymous IJCAI and workshop reviewers for their thorough and insightful feedback. 


\section{References}

[Aziz et al., 2017] Haris Aziz, Markus Brill, Vincent Conitzer, Edith Elkind, Rupert Freeman, and Toby Walsh. Justified representation in approval-based committee voting. Social Choice and Welfare, 48(2):461-485, 2017.

[Aziz et al., 2018] Haris Aziz, Edith Elkind, Shenwei Huang, Martin Lackner, Luis Sánchez-Fernández, and Piotr Skowron. On the complexity of extended and proportional justified representation. In Proceedings of the 32nd AAAI Conference on Artificial Intelligence, pages 902909, 2018.

[Brams et al., 2007] Steven J Brams, D Marc Kilgour, and M Remzi Sanver. A minimax procedure for electing committees. Public Choice, 132(3-4):401-420, 2007.

[Brandl and Peters, 2019] Florian Brandl and Dominik Peters. An axiomatic characterization of the Borda mean rule. Social Choice and Welfare, 52(4):685-707, 2019.

[Brill et al., 2017] Markus Brill, Rupert Freeman, Svante Janson, and Martin Lackner. Phragmén's voting methods and justified representation. In Proceedings of the 31st AAAI Conference on Artificial Intelligence, pages 406413, 2017.

[Cheng et al., 2019] Yu Cheng, Zhihao Jiang, Kamesh Munagala, and Kangning Wang. Group fairness in committee selection. In Proceedings of the 20th ACM Conference on Economics and Computation (EC), pages 263-279, 2019.

[Duddy et al., 2016] Conal Duddy, Ashley Piggins, and William S Zwicker. Aggregation of binary evaluations: a Borda-like approach. Social Choice and Welfare, 46(2):301-333, 2016.

[Faliszewski et al., 2017] Piotr Faliszewski, Arkadii Slinko, and Nimrod Talmon. The complexity of multiwinner voting rules with variable number of winners. arXiv preprint arXiv:1711.06641, 2017.

[Fishburn and Pekeč, 2004] Peter C Fishburn and Aleksandar Pekeč. Approval voting for committees: Threshold approaches. Technical Report, 2004.

[Janson, 2016] Svante Janson. Phragmén's and Thiele's election methods. arXiv preprint arXiv:1611.08826, 2016.

[Kilgour and Marshall, 2012] D Marc Kilgour and Erica Marshall. Approval balloting for fixed-size committees. In Electoral Systems, pages 305-326. 2012.

[Kilgour et al., 2006] D Marc Kilgour, Steven J Brams, and M Remzi Sanver. How to elect a representative committee using approval balloting. In Mathematics and Democracy, pages 83-95. Springer, 2006.

[Kilgour, 2016] D Marc Kilgour. Approval elections with a variable number of winners. Theory and Decision, 81(2):199-211, 2016.

[Mueller, 1969] John E Mueller. Voting on the propositions: Ballot patterns and historical trends in california. American Political Science Review, 63(4):1197-1212, 1969.
[Peters and Skowron, 2019] Dominik Peters and Piotr Skowron. Proportionality and the limits of welfarism. arXiv preprint arXiv:1911.11747, 2019.

[Phragmén, 1899] Edvard Phragmén. Till frågan om en proportionell valmetod. Statsvetenskaplig Tidskrift, 2(2):297305, 1899.

[Sánchez-Fernández et al., 2017] Luis Sánchez-Fernández, Edith Elkind, Martin Lackner, Norberto Fernández, Jesús A Fisteus, Pablo Basanta Val, and Piotr Skowron. Proportional justified representation. In Proceedings of the 31st AAAI Conference on Artificial Intelligence, pages 670-676, 2017.

[Skowron et al., 2017a] Piotr Skowron, Martin Lackner, Markus Brill, Dominik Peters, and Edith Elkind. Proportional rankings. In Proceedings of the 26th International Joint Conference on Artificial Intelligence (IJCAI), pages 409-415, 2017.

[Skowron et al., 2017b] Piotr Skowron, Martin Lackner, Edith Elkind, and Luis Sánchez-Fernández. Optimal average satisfaction and extended justified representation in polynomial time. arXiv preprint arXiv:1704.00293, 2017.

[Skowron, 2018] Piotr Skowron. Proportionality degree of multiwinner rules. arXiv preprint arXiv:1810.08799, 2018.

[Thiele, 1895] Thorvald N Thiele. Om flerfoldsvalg. Oversigt over det Kongelige Danske Videnskabernes Selskabs Forhandlinger, 1895:415-441, 1895. 